\title{
A Conceptual Model to Explain Dark Matter and Dark Energy
}

\author{
Jonathan Blackledge \\ Honorary Professor, School of Mathematics, Statistics and Computer Science, \\ University of KwaZulu-Natal, South Africa. \\ Honorary Professor, School of Electrical and Electronic Engineering, \\ Technological University Dublin, Ireland. \\ Visiting Professor, Faculty of Arts, Science and Technology, \\ Wrexham Glyndwr University of Wales, UK. \\ Distinguished Professor, Centre for Advanced Studies, \\ Warsaw University of Technology. \\ Professor Extraordinaire, Faculty of Natural Science, \\ University of Western Cape, South Africa. \\ Stokes Professor, Science Foundation Ireland. \\ Email: jonathan.blackledge@TUDublin.ie
}

\begin{abstract}
This paper considers a conceptual model that attempts to explain 'Dark Matter' and 'Dark Energy'. The model is based on considering a gravitational field to be the result of a mass (a Higgs field) scattering pre-existing cosmic background space-time waves or 'Uber-waves'. The term 'Uber' is used to denote an outstanding or supreme example of a particular kind of gravitational wave with cosmic-scale wavelengths that are far in excess of those associated with the gravitational waves generated by accelerating masses. Such waves are taken to be the very lowest frequency components associated with the spectrum of space-time waves generated by the 'Big Bang' and are supported by the expanding fabric of space-time produced at the point of the big bang, i.e. the lowest frequency components of a cosmological spectrum whose bandwidth is the a Planck frequency $\left(\sim 10^{43} \mathrm{~Hz}\right)$. Like electromagnetic waves, Uber waves are taken to propagate with an upper velocity consistent with the speed of light and interact with, and are scattered by, a Higgs field. This interaction produces the effect of a mass locally curving space-time, an idea that is contrary to the conventional model associated with General Relativity where mass is taken to curve space-time directly which otherwise remains 'flat'. By assuming the pre-existence of background Uber waves, we consider the concave curvature of such waves to generate an apparent attractive gravitational force. This attractive force is taken to govern the formation of large scale structures of matter (galaxies and super-clusters of galaxies, for example) in the conventional sense but surrounded by a residual background gravitational field. It is this residual field that gives rise to the effect known as dark matter where more gravity (as an attractive only force) appears to be available than that which can be accounted for by the observed (luminous) mass, a luminosity that is generated primarily by nuclear fusion in stars. The convex curvature of Uber waves is considered to account for cosmic voids within which gravity is a repulsive force and where large scale structures of matter can therefore not be formed. This is considered to explain the super-large cosmic voids or super voids that are observed. These are regions of the universe where there is an absence of rich super clusters of matter. In these anti-gravity zones, only relatively small structures of matter can be formed by electrostatic forces alone which are then repelled from each other when their mass becomes significant enough for the force of anti-gravity to become significant. In such regions of an Uber wave, the matter generated from electrostatic forces builds up to produce a weak gravitational repulsive field due to the low mass density within a void. However, due to the immense size of these cosmic voids, they are taken to generate a net repulsive force which is considered to be the reason for the acceleration associated with the expansion of the universe; the effect of dark energy. This effect also accounts for the cosmic web structure in which luminescent matter appears to exist in relatively thin connective filaments. The purpose of this paper is to provide a conceptual model and not to investigate the ideas proposed in any significant mathematical detail. This is accomplished by building up the ideas on a case-by-case basis, coupled with a series of thought experiments but without resorting to specific physical scales or the physical parameters associated with these scales other than, by default, the speed of light and Newton's gravitational constant.
\end{abstract}

Keywords: gravitational waves, Uber (space-time) waves, dark matter, dark energy. 


\section{Introduction}

Dark Matter and Dark Energy are terms that exist in name only. This is because to date, there is no accepted physical understanding and associated physical model(s) that explains what these terms really mean. However, it is currently excepted that the physical effects these terms attempt to describe are real physical observables. While this paper does not claim in any way to have solved the problem of what dark mattar and dark energy actually are, the ideas and conceptual model that is introduced are considered to be an original contribution in an attempt to obtain a possible solution. In this context, the introduction now provided gives a brief overview of the terms dark matter and dark energy in order to give a background to the conceptual ideas that are presented. This includes a short overview on some of the principal theoretical concepts that are currently being considered.

\subsection{Dark Matter}

The term dark matter was first coined by Fritz Zwicky in 1933 [1]. While studying a galaxy cluster, he observed that the rotational velocity of the cluster required much greater gravitational forces of attraction to be present than could be accounted for by the combined mass of the 'luminous matter', i.e. matter that can be seen from the light (and other electromagnetic radiation) emitted by stars undergoing a process due to the strong force - the process of nuclear fusion which is a result of the immense gravitational force generated by the extent of the mass of a star. Zwicky therefore inferred that the extra gravitational force required to account for the structure and dynamical characteristics of the cluster must be due to some non-luminescent mass or 'dark matter' [2].

Although the existence of dark matter is now an accepted idea, the physics associated with this concept remains hypothetical. Nevertheless, it is now taken to account for over $80 \%$ of the matter in the universe. Commonly considered to be composed of some, as-yet, undiscovered sub-atomic particle, to-date, dark matter remains a physical phenomenon in name only, with no accepted satisfactory explanation, nearly 100 years after it was first conceived.

The expansion of the universe was first observed in the late 1920s through the work of Edwin Hubble and was based on the correlation between the distance of a galaxy and its red shift [3]. This provided support for what later became known as the big bang model, originally conceived by Georges Henri Lemaitre in 1927 [4] through an interpretation of Einstein's model for gravity, i.e. the Theory of General Relativity [5].

The motion or 'Hubble Flow' of astronomical objects is taken to be due solely to this expansion and is quantified by Hubble's law. This law relates the velocity of expansion $v$ to the 'proper distance' $D$ (which can change over time) of a galaxy by the simple linear equation $v=H D$ where $H$ is the Hubble constant, usually expressed in terms of the 'Hubble time' given by $H^{-1} \sim 5 \times 10^{17} \mathrm{~s}$.

\subsection{Dark Energy}

The term, dark energy, describes an unknown form of energy that was proposed in 1998 to account for an observation (based on measuring the distance of supernova events) which is that the expansion of the universe does not expand at a constant rate according to Hubble's law but is rather undergoing an acceleration [6]. In this context, dark energy is considered to be a repulsive gravitational force (at least within the space it occupies) that is a dominant component $(\sim 70 \%)$ of the universe, the remaining portion of the universe consisting of ordinary matter and dark matter. However, like dark matter, dark energy remains speculative with no accepted physical explanation to date.

What ever dark energy actually is, it is considered to be of very low density and highly homogenous, interacting only with one of the four fundamental forces of nature, namely, Gravity. Dark energy is considered to be a cosmic repulsive gravitational force, a concept that was actually first hypothesised by Einstein in 1917 and compounded in a term which he introduced into his gravitational field equations and is called the 'Cosmological Constant' [7]. However, the reason he introduced this constant was to counteract the effect of gravity being an attractive-only force and because, at the time, he and the scientific community considered the universe to be static (neither expanding or contracting). If gravity was taken to be an attractive only force, then the universe would collapse and so the cosmological constant was 
invented by Einstein to provide a static universe model. The idea was abandoned when Hubble observed the universe to be expending, but since this expansion is now known to be accelerating, the idea of a repulsive gravitational effect, and a dominant one at that, is back in vogue.

\subsection{Current Theoretical Concepts}

While there is currently no unified and universally accepted theory for explaining dark matter and dark energy, there has been and continues to be a significant amount of effort to realising the physics associated with these phenomena. One of the underlying problems is that dark matter has yet to be observed directly while dark energy is a concept that is currently taken to account for the observed acceleration in the expansion of the universe. It is clear that dark matter, whatever it is, must only very weakly interact (if at all) with ordinary matter and radiation; hence, the reason for its non-observability. For this reason models for dark matter tend to be based on the existence of an elementary particle or class of particles that have yet to be discovered. Such particles are considered to be massive particles that only interact weakly with ordinary matter particles or Baryons. For this reason, they are known as Weakly Interacting Massive Particles (WIMPS) [8], [9] for which experimental work has and continues to be actively undertaken in regard to their possible future detection, e.g. [10] - [15]. Another approach to explaining dark matter is based on considering modifications to the theory of general relativity that may be able to account for all observations without invoking supplemental non-baryonic matter [16]. This includes models based on the idea of multi-cohesive areas, for example [23].

Efforts to explain dark matter/energy can, in principle, be considered within the framework of extended gravity cosmography, in which the theory of general relativity is taken to be a special case of an 'extended' theory [17], [18]. This is because the observations that the universe has entered an accelerated phase cannot be explained alone by the 'dynamics' of ordinary matter and radiation as constituents of a 'Cosmic Fluid' with a flat spatial curvature as suggested from observations of 'Cosmic Microwave Background Anisotropies' [19]. Within the framework of the theory of general relativity, one of the simplest explanations for the accelerated expansion of the universe is the introduction or re-introduction (from Einstein's original proposal [20]) of a cosmological constant' [21] which defines the concordance of a 'Cold Dark Matter' model [22]. However, coupled with the general development and cosmological applications of the theory of gravity, there are numerous models that have and continue to be proposed, many of which attempt to unify the physics associated with both dark matter and dark energy. Such models continue to be developed and published in a range of journals and special issues such as those considered in [24] and [25], respectively, for example.

\section{Propagative Models of Gravity: A Short History}

The conceptual model considered in this paper has a synergy with the so called mechanical and propagative wave theories of gravity which have an interesting history. These were ideas conceived in an attempt to explain why a gravitational force exists instead of just accepting how it operates according to Newton's law of gravity. However, they were effectively abandoned following the success of Einstein's theory of general relativity in 1916, and, in particular, its experimental verification by Eddington in 1919 through the observation of the deflection of light by the gravitational field generated by the sun [26], [27]. This experimental verification was actually coupled with Eddington's less than purely scientific adoption of the theory for personal reasons [28]. However, it is now an accepted that Einstein's field equations provide the most accurate model for gravity known to date.

The mechanical theory of gravity was an original idea attributed to Nicolas Fatio de Duillier in 1690 and was re-invented by Georges-Louis Le Sage in 1748, Lord Kelvin in 1872 and Hendrik Lorentz in 1900. The fundamental problem associated with this concept was why a mass does not heat up through the absorption of a universal field of particles which was taken to generate the force of gravity by pushing two masses together.

The origins of the propagative theory of gravity is attributed to Pierre-Simon Laplace in 1805 when he considered the so called 'Speed of Gravity' [29]. The underlying drive for this development was to overcome a fundamental problem with Newton's law, namely, the principle of instantaneous action at 
a distance in which Newton's gravitational force is taken to be 'transmitted' instantaneously. Laplace assumed the propagation of a wave field propagating at some finite speed, originating from a source - a mass. A gravitational force was then attributed to some type of interaction of this wave field with another source which generated a propagating wave field itself. This has an synergy with, but is not the same,as, the concept presented in this paper. However, in this paper the wave field is taken to a back-ground low frequency space-time wave which interacts with both sources simultaneously. In this context, a principle problem with Laplace's idea was that gravitational attraction is towards the instantaneous position of a mass with no time delay; it is not a time retarded effect which must be considered to be an intrinsic property of any finite propagative interaction.

In the latter part of the nineteenth century, eminent physicists including Weber, Riemann, Maxwell and others attempted to advanced Laplace's ideas by introducing corrective terms. For example, Maurice Levy in 1890 and Paul Gerber in 1898 used the finite propagation theory of gravity to explain the perihelion shift of Mercury's orbit. These concepts were in effect, attempts to 'engineer' a theory of gravity based Newtonian mechanics with an idea first introduced by Laplace. But it was an approach that had passed its sell-by date, thereby setting the scene for the universe according to Albert Einstein. They were concepts based on some source-to-source interaction.

The concept considered in this paper is different because it is predicated on the propagation of pre-existing gravitational Uber waves. In this case, space-time is not flat in the absence of a mass but supports ultra-low frequency space-time waves a priori. Thus, the essential conceptual difference between the idea developed by Laplace et al. and the one presented in this work, is that a gravitational field is not due to the effect of some point-to-point source propagation effect in a flat three-dimensional space but due to the scattering by such point sources of pre-existing space-time waves generated by the big bang. In this sense a gravitational field is the by-product of the scattering of an ultra-low frequency space-time wave with a Higgs field.

\section{Overview of the Concept}

This paper attempts to give an explanation for both dark matter and dark energy as manifestations of the same physical concept. The origins of the concept proposed comes from asking the question, why does matter curve space-time? While the curvature of space-time by a mass is the central kernel of Einstein's model for gravity, there is no deeper explanation as to why this should be the case. It is a manifestation of a phenomenology, which is well stated by John Wheeler in terms of the following: 'Space-time tells matter how to move and matter tells space-time how to curve' [30]. In this sense, while Einstein's model is a monumental, if, non-intuitive improvement to Newton's model, it still suffers from the same basic issue; the basis for a criticism of Newton's model in his day, to which Newton replied: 'I make no hypotheses .... I have told you how it works, not why it works'. The question is therefore, why does matter tell space time how to curve and who exactly is telling who! If we consider the fabric of space-time to be an ultra-low frequency wave and not a flat structure in the absence of mass, then although matter will still tell space-time how to curve in the conventional way, because of the wave structure of space-time that is assumed a priori, the curvature of space-time can be then be taken to be due to a scattering interaction with the mass, albeit at a wavelength whose scale length is vast compared to that of a mass, at least when the mass is taken to be of compact support.

Even with the recent discovery of the Higgs Boson to explain the concept of mass [31], it is still not fully understood why a Higgs field curves space-time. We therefore consider the case where mass does not of itself curve space-time; rather it is an apparent effect which is a consequence of the mass interacting with pre-existing ultra-low frequency and ultra-long wavelength space-time waves or 'Uber waves', a term that is used to denote a supreme (low-frequency) example of a gravitational wave. The interaction is taken to produce a scattered space-time wave field but at a frequency that is so low that the scattered field appears to have no oscillation over the scale of a galaxy cluster, for example and beyond and is therefore perceived to be a static field in the sense that there are no oscillation in the local vicinity of the interaction. Only the spatial characteristics of the scattered wave field then remain, leading to a (weak) gravitational force being determined by the inverse square law.

In the context of this idea, the Uber waves which generate gravitational forces (both attractive and repulsive) are taken to be a consequence of the big bang. They represent the very lowest frequency 
components associated with the spectrum of waves generated by this event and are supported by an expanding space-time manifold. These waves consist of cycles in which space-time is concave and convex, analogous to the the negative and positive amplitude components associated with a conventional cosinusoidal wave, for example. A concave curvature generates an attractive gravitational potential and a convex curvature produces a repulsive potential. Thus, the concave space-time of such waves generates conventional gravitational forces where gravitation is an attractive-only force governing the formation of large-scale structures of matter such as galaxies and clusters of galaxies. However, the scale length of such structures is insignificant compared to the background gravitational potential associated with the larger-scale space-time concave curvature in which such structure have been created and develop over time. The background curvature associated with the concave curvature of an Uber wave, where gravity manifests itself as an attractive-only force, is then taken to explain dark matter, i.e. an explanation as to why there appears to be more gravity than the mass that can be observed.

In the context of the above, the convex curvature of such space-time Uber waves are regions where gravity is a repulsive-only force and where large structures of matter can therefore not develop. In these regions of the universe, matter can coalesce and grow in size and mass through electrostatic forces when the weak force of gravity is insignificant as is the case in either region (concave and convex). However, when these clumps of matter become large enough to have a mass that makes a gravitational force significant, gravity pushes them away from each other instead of attracting them together to produce increasingly larger bodies of matter giving rise to stars, galaxies and galaxy clusters. This effect is taken to account for the cosmic voids that are observed in the universe, where a conventional distribution of matter, assessed through a lack of luminosity, appears to be missing.

At the interface between the transitions from a concave to a convex space-time curvature of an Uber wave, space-time is flat and a gravitational force does not exist (attractive or repulsive). In the convex curvature of the Uber wave, as matter builds up through electrostatic forces alone, a weak gravitational repulsive potential develops due to the very low and relatively homogenous mass density within a cosmic void. The net effect of this is to produce vast regions of anti-gravity (the cosmic voids) which push against the dark matter components of the universe (cosmic regions composed of ordinary matter) thereby accelerating the expansion of the universe as a whole and accounting for an observation in which ordinary matter (at least in a luminescent state) appears to 'fly apart' at an ever increasing speed. It is this effect that is taken to be described by the term dark energy.

The purpose of this paper is to implant an 'image' into the mind of the reader of how the conceptual model proposed above might explain dark matter and dark energy without having to resort to a detailed mathematical description and introducing specific physical scale lengths and/or associated physical constants. In this respect, the approach taken is entirely qualitative and relies on a descriptive narrative coupled with schematic graphical representations and a certain amount of repetition to emphasise key points. However, from time to time, the author has considered it to be of value to introduce certain mathematical descriptors in order to quantify some specific issues. These 'descriptor's' are based on a scalar field theory, and, in this context, uses a semi-Newtonian approach that is representative of a weak field approximation to model a gravitational field based on the general theory of relativity, i.e. the case when Einstein's correction to the Newtonian model reduces back to the Newtonian model. The principal idea is developed on the case-by-case basis given in the following section which is followed by and coupled with a sequence of thought experiments. In both cases, the formulation is entirely deductive and non-empirical.

\section{A Case-by-Case Explanation of the Concept}

In the explanations that follow, we work within the back-ground context of a linearised version of Einstein's equations for a gravitational field. The linear form of these equations are a consequence of applying a weak (gravitational) field condition when the curvature of space-time becomes a small perturbation of an otherwise 'flat space-time' so that there is minimal curvature in the fabric of space-time. This approximation describes a 'Minkowski space' that, even for a curved space-time, provides a good description in an infinitesimal region surrounding any point, barring gravitational singularities. In this case, Einstein's equations reduce to a wave equation representation for each component of space-time. This equation is linear and characterised by the classical d'Alembertian wave operator. It allows the concepts 
presented to be appreciated within the context of gravitational waves, the use of a linearised gravitational field being integral to the study of such waves and weak-field gravitational lensing, for example. A linear gravitational wave includes components that are tangential to the direction of propagation but also include a component in the direction of propagation analogous to pressure waves in acoustics, for example.

Linear gravitational wave theory provides the background to modelling gravitational waves generated by a source - a mass undergoing an acceleration. Here, we consider such waves to have a wavelength on a much larger cosmological scale and to have been generated by the big bang prior to the development of stars and galaxies, for example. They are supported by the fabric of space-time, a fabric that is taken to be expanding at a constant rate according to a Hubble type law as discussed in Section 1.1. This linearised approach actually leads to an underlying inconsistency which is addressed later on in the paper. However, it is within this linearisation, that the following explanation of the concept is now delivered on a case-by-case basis.

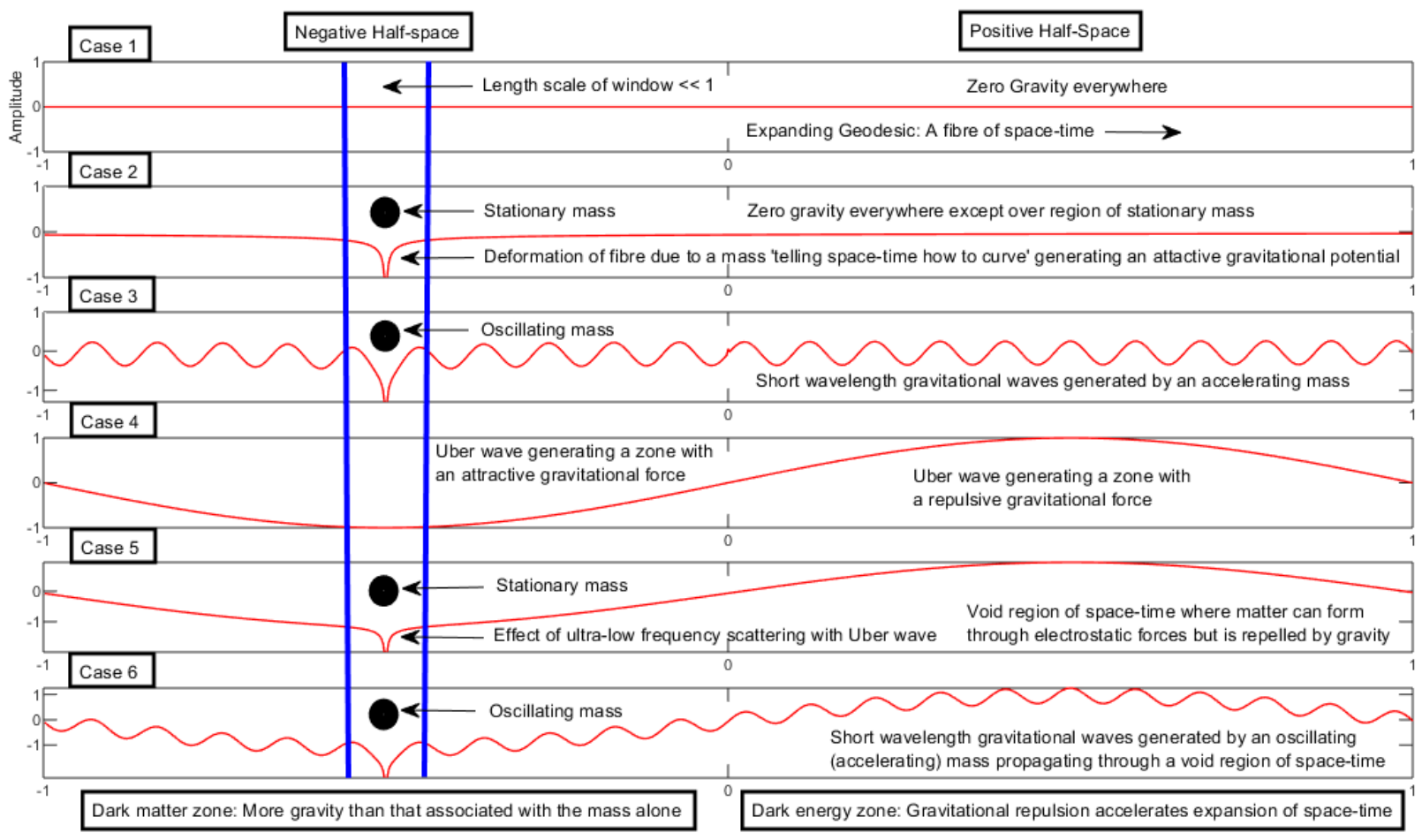

Figure 1. Schematic illustration referencing the 'Cases' associated with a description of the conceptual model for dark matter and dark energy.

Consider Figure 1 which shows six 'Cases' whose aim is to conceptually distinguish between the conventional model for the generation of a gravitational field (Cases 1-3) and the Uber wave model being considered (Cases 4-6). The figure considers a negative and a positive half-space in the range -1 to +1 and an 'amplitude' over the same scale. This is by convention only and no physical significance is to be placed on the numerical range and scales given. However, a negative amplitude is taken to represent a gravitational field that produces an attractive force between two masses. Similarly, a positive amplitude is taken to represents a gravitational field that produces a repulsive force between to masses. The difference in the polarity of the amplitude to differentiate between an attractive or repulsive gravitational field is by convention alone; it represents the difference between space-time having a weak concave curvature (producing an attractive force) or a weak convex curvature (producing a repulsive force), respectively. In this sense, the negative and positive half-spaces given Figure 1 (Cases 4-6) represent a single cycle of an Uber wave and (from left to right) its transition from a concave to a convex space-time. The figure includes a window (within the blue vertical given) whose physical scale length is taken to be insignificantly small 
compared to that which lies out side the window (to both the left and right hand side of the window). No significance should be attributed to the fact that this window has been placed in the mid-point of the negative half-space.

\subsection{Case 1}

Consider a universe in which there is no matter and no gravity (and no conventional gravitational waves). All that exists is a fabric of space-time which is 'flat' as represented by the line or string with zero amplitude - a so called 'fibre' of space-time (colour coded in red). This line represents a Geodesic in space-time which is taken to be have expanded and continues to do so expanding as a result of the big bang. This expansion is assumed to have a constant velocity and no rate of change in this velocity according to the Hubble model. This figure is a one-dimensional analogy of a flat space-time which is more conventionally represented graphically by a uniform (flat) surface.

\subsection{Case 2}

Within the window shown in Figure 1, where the physical scale in much less than 1 and is colour coded in blue, let a stationary mass be introduced as illustrated. The mass is taken to be stationary within the reference frame given. According to Einstein's model of gravity, this mass curves the space-time that surrounds it generating an attractive gravitational potential. The force between this mass and that of another body is determined by the gradient of this potential and not the potential itself. In Figure 1, this is represented by a change in the amplitude of the Geodesic from 0 to negative values over the region of space within the vicinity of the mass so that by convention, curvature of the space-time fibre with an amplitude that is less than zero produces an attractive gravitational force. The negative value is representative and only representative of gravity being an attractive force. This representation of space-time curvature is taken to be the product of a weak gravitational field generated by a relative small mass in cosmological terms and not an object such as a black hole, for example, which generates a gravitational singularity.

\subsection{Case 3}

In this case, the mass is taken to accelerate through some oscillation (acceleration). A proportion of energy of the oscillation is converted into gravitational waves with some relatively short wavelength $\lambda$ (on a cosmological scale) which propagate at the speed of light and whose energy dissipation conforms to the inverse square law. These waves propagate away from the mass generating ripples in the flat 'fabric of space-time', represented by the rippling of the Geodesic as illustrated in Figure 1, 'Case 3'. The gravitational waves are taken to be examples of those that were first recorded on September 14, 2015 [32] confirming the original proposal by Henri Poincaré in 1905 [33] and subsequently predicted by Albert Einstein in 1918 [34], [35]. In reality, such waves are not generated by a simple oscillating mass as inferred from the schematic given in Figure 1 (Case 3). In the first 2015 observation, they were produced by the equivalent effect of an oscillation in terms of a process undergoing an acceleration, but where this process involved the evolution of a coalescing binary system (consisting of two Black Holes). As the orbital period of the in-spiral binary system reduced, the energy of the gravitational waves emitted increased as did their frequency, thereby generating a gravitational wave with the signature of a 'Chirplet' (a time limited pulse over which a frequency modulation occurs), e.g. [36].

\subsection{Discussion}

The explanation of Cases 1-3 given in Figure 1 conform to a conventional model of gravity compounded in Einstein's theory of general relativity under the weak field approximation. Here, a stationary mass curves space-time and an oscillating mass generates gravitational waves. Note however, that the generation of such gravitational waves is predicated on the curvature of space-time by a mass and that the concept illustrated in Case 2 preempts the effect illustrated in Case 3. But the concept illustrated in Case 2 is not explained, namely, why does a mass curve space-time? In an attempt to explain the effect illustrated 
in Case 2, we reverse the roles of an oscillating mass and the production of gravitational waves by considering a pre-existing ultra-low frequency ultra-long wavelength gravitational wave (an Uber wave) with a cosmologically long wavelength $\Lambda>>\lambda$ that interacts with a mass to produce the gravitational field. This idea is described in Cases 4-6 below in reference to the schematic illustrations given in Figure 1.

\subsection{Case 4}

Consider a universe in which there is no matter and no gravity (due to the presence of a mass in the conventional sense) but where there are Uber (space-time) waves as illustrated by the single cycle wave form given in Figure 1, Case 4. No inference is implied in this figure as to the physical extent of the waves, their periodicity or type (i.e. free or standing waves). They are taken to exist as small perturbations to an otherwise flat space-time fabric as illustrated in Case 1 and are therefore an approximation to Einstein's field equations under the weak field condition. In this context, the scale of the amplitude in the figure is only a representative scale and not of any physical significance.

Such waves are taken to have been created at the point of the big bang. They are a manifestation of the lower frequency spectrum generated by this event, supported by the expanding fabric of space-time. Although represented in the figure as a scalar one-dimensional wave with a single cycle, it should be understood that this is a representation of a cosmic scale wave with many cycles and is a tensor field that compresses and stretches space-time as it propagates at light speed. The negative half-space generates an attractive gravitational force (in the conventional sense) but such a force only becomes apparent if at least two point masses are present. The positive half-space generates the potential for a repulsive gravitational force if at least two large enough masses were to exists. No inference on the gravitational strength should be inferred from the changing wave amplitude illustrated, Newton's gravitational constant being taken to be the same in both the negative and positive half-spaces.

\subsection{Case 5}

Let a mass be introduced within the window shown in Figure 1 whose physical scale is much less than 1. This mass interacts with the back-ground space-time Uber waves, an interaction that produces the same effect as given in Case 2. However, in this case, the curvature of space-time over the locailty of the mass is due to a scattering interaction where the frequency of the interaction is so low as to manifest itself as an apparent static field - a conventional gravitational field. The generation of this field is taken to conform to the Source-Scattering Equivalence Principle to be discussed in Section 5.

Let us now consider the case when a very low but homogenous mass density is introduced to both the negative and positive half-spaces. In the negative half-space, material starts to clump together through attractive electrostatic forces coalescing dust and gas. As the mass of this material increases, a point is reached when gravitational attraction can start to influence this material to form larger and larger structures leading to planets, stars, galaxies, galaxies clusters and super clusters etc. The observation that the dynamic behaviour of such clusters requires much more mass (dark matter) than can be accounted for from their luminosity is then explained by the existence of the back-ground residual gravitational potential represented by the negativity (i.e. the concave curvature) of the Uber wave amplitude (between -1 and 0 , representative).

In the positive half-space, while material can start to clump together through electrostatic forces, the evolution of matter in the negative half-space through gravitational attraction is not possible because gravity is a repulsive force. The positive half-space therefore represents regions of the universe that are cosmically void of matter, i.e. planets, stars and galaxies and galaxy clusters can not exist. Nevertheless, the relatively homogenous distribution matter in these cosmic voids represents a large repulsive gravitational force. The positive half space therefore pushes on the negative half-space increasing the velocity at which the Geodesic is observed to expand, thereby providing a possible explanation for the increasing acceleration of the universe.

\subsection{Case 6}

As in Case 3, an oscillating mass generates gravitational waves with a relatively short wavelength $\lambda$. These waves propagate away from the mass at the speed of light but now they generate ripples in the 
fabric of a much longer wavelength Uber wave. In Figure 1 (Case 6), this is represented by the rippling of the Uber wave amplitude, a cosmological wave that is ultimately responsible for the creation of the luminous matter in the first place through the argument given in Case 5.

\subsection{Discussion}

In Case 5, we consider the initial mass densities in both the positive and negative half-spaces to be the same which then undergoes a very different evolution in time. In both regions, attractive electrostatic forces are taken to form small clumps of matter with a mass density that is initially too small for gravitational forces to be effective. When the mass of this matter become large enough, gravity takes over to form visible structures in the negative half-space (through the evolution of stars by gravitational attraction) and invisible structures in the positive half-space due to the effect of gravitational repulsion. In both half-spaces, the physics of the electromagnetic force, the weak force and the strong force are considered to be identical. Virtual particles are taken to be created and annihilated in equal measure. In the positive half space, however, it is not possible to generate a black hole, for example, and so the generation of Hawking radiation [45] through virtual particle dissipation at the event horizon of a black hole is not possible. In both half-spaces, a gravitational field is not just the effect of a mass warping space-time but the result of a scattering interaction with an existing space-time Uber wave. A scattering effect is not possible if space-time is taken to be flat as in Figure 1, Case 1. Thus, the assumed existence of Uber waves can, through the ideas expressed in Figure 1, help to explain not only dark matter and dark energy but the reason for the generation of a gravitation field through a fundamental interaction between a space-time wave field and a Higgs field. An important feature of this concept is quantified in the equivalence principle as discussed in the following section.

\section{Source-Scattering Equivalence Principle in the Zero Frequency Limit}

The equivalence principle considered in this section relates to the inability to distinguish between the gradient of a wave field generated by a source and the scattering of a wave field by the same source in the limit as the frequency of the field approaches zero. It is assumed that the source producing the wave field can itself be oscillated at an identical frequency by a wave field that is generated independently of and externally to the source. In the following sections, we consider some basic properties of such waves using a model based on three-dimensional scalar wave functions.

\subsection{Wave Field Generated by a Point Source}

Ignoring the physical characteristics of a source and associated waves, let us assume the generic case, when energy is applied to some (otherwise stationary) point source which undergoes an oscillation. A proportion of this energy is then converted into wave motion, a conversion that is assumed can be achieved at any frequency. For a point source oscillating at angular frequency $\omega$ say, the pattern of the three-dimensional waves (the three-dimensional scalar 'wave field' $\phi$ ) is given by

$$
\phi(\mathbf{r}, k)=G \frac{\exp (i k r)}{r}
$$

where $\mathbf{r}$ is the three-dimensional space vector, $r=|\mathbf{r}|$ is the distance of the wave amplitude from the source, $k=\omega / c_{0}$ where $c_{0} \simeq 3 \times 10^{8} \mathrm{~ms}^{-1}$ is the speed of light and $G$ is some constant which determines the energy conversion from the source to the wave field.

The intensity of this 'out-going' wave field (a measure of its energy) is given by

$$
|\phi(\mathbf{r}, k)|^{2}=\left|G \frac{\exp (i k r)}{r}\right|^{2}=\frac{G^{2}}{r^{2}},
$$

an energy distribution that conforms to the inverse square law. Here, the frequency of the wave field is taken to be determined exactly by the oscillation of the source. 
Suppose we now reduce the frequency of oscillation of the source so that the frequency of the wave field is reduced proportionately. As the frequency reduces to zero, the wave field become characterised by $G / r$ alone given that $\exp (i k r)$ reduces to 1 . Thus, for the case when a single point source generates a wave field

$$
\phi(\mathbf{r}, k)=\frac{G}{r}, k \rightarrow 0,
$$

The energy of such a near zero frequency wave field again conforms to the inverse square law. This is the case for a point source producing an ultra-low frequency wave field.

\subsection{Wave Field Generated by a Point Scatterer}

Now suppose that the source is stationary, but that a (unit amplitude) plane wave denoted by $\phi_{i}(\mathbf{r}, k)=$ $\exp (i \mathbf{k} \cdot \mathbf{r})$ is incident upon the source where $\mathbf{k}$ is the spatial frequency vector. The source of this plane wave is taken to be from an origin that is external too, and, independent of the source.

Just as the source can produce waves through oscillation, the same source is now taken to be oscillated by an incident wave for which we again assume a one-to-one correlation in frequency between that of the wave and the source. But if the source oscillates, then it must itself produce a wave field, thereby providing consistency with the scenario given in Section 5.1. This is the 'scattered wave field' generated by the interaction of the wave field with the point source.

In this case, the entire wave field is the sum of the incident and scattered wave fields and we can therefore express this wave field in the form

$$
\phi(\mathbf{r}, k)=\phi_{i}(\mathbf{r}, k)+G \frac{\exp (i k r)}{r}
$$

The intensity of the wave field is therefore

$$
\begin{gathered}
|\phi(\mathbf{r}, k)|^{2}=\left|\phi_{i}(\mathbf{r}, k)+G \frac{\exp (i k r)}{r}\right|^{2}=\left[\phi_{i}(\mathbf{r}, k)+G \frac{\exp (i k r)}{r}\right]\left[\phi_{i}(\mathbf{r}, k)+G \frac{\exp (i k r)}{r}\right]^{*} \\
=1+\frac{G^{2}}{r^{2}}+\frac{G}{r}\left[\phi_{i}^{*}(\mathbf{r}, k) \exp (i k r)+\phi_{i}(\mathbf{r}, k) \exp (-i k r)\right]
\end{gathered}
$$

This is a very different result to the wave field generated by a source alone because of the cross terms. Note however, that if the frequency reduces to zero, then the wave field amplitude $\phi$ reduces to the form $1+G / r$. In this context, the difference between a wave field generated by an oscillating source and the wave field produced by the interaction with an incident plane wave is compounded in the addition of a constant. In this analysis, the constant is 1 because we have assumed that the incident plane wave field has a unit amplitude. These results are quantified in the following:

Wave field generated by a point source:

$$
\phi(\mathbf{r}, k)=\frac{G}{r}, k \rightarrow 0
$$

Wave field generated by a point scatterer interacting with a (unit amplitude) plane wave:

$$
\phi(\mathbf{r}, k)=1+\frac{G}{r}, k \rightarrow 0
$$

\subsection{Low Frequency Field Gradient Equivalence}

Suppose that an 'observer' of the wave field $\phi$ can only 'measure' the gradient of the field where the field is conservative and irrotational. In the ultra low-frequency limit, they will not observe any difference between the wave field that has been produced by a point source and the wave field generated by the interaction of an existing wave field with the same point 'source'. This is because the gradient of $G / r$ is identical to the gradient of $1+G / r$. In this sense, one can not determine whether the source of the field 
(i.e. the gradient of the ultra-low frequency wave field) is being generated directly or indirectly by the source.

The idea here is that for an isolated single point scatterer, the field it produces through its interaction with background ultra-long wavelength waves can only be measured through the effect it has on another point scatterer. This is because it is the gradient of such a field that is responsible for generating an acceleration (a measurable effect due to a force) and not the field itself. Until the field generated by one source is 'measured' through an interaction with another, the field does not effectively exist. Only when another point source (which is taken to generate the same low frequency wave field by scattering the same Uber wave) is present, will the field become measurable. But this measure is only possible through the gradient of the field because it is the gradient that generates a measurable effect.

\subsection{Exact Born Scattering}

If $G<<1$, then the scattering is a weak effect given that the scattered wave field is a small perturbation of the incident field $\phi_{i}(\mathbf{r}, k)$ and $\|\phi\| \sim \mid \phi_{i} \|$. This is known as the weak scattering condition and for $G<<1$, the model conforms to the Born approximation [37]. In general, this approximation requires that the wavelength of the incident wave field is much larger than the physical scale of the scatterer as is consistent with the schematic given in Figure 1 (Case 5). The Born approximation is fundamental to the deterministic modelling of scattered wave fields, an approximation which becomes exact in the limit as the frequency tends to zero. Thus, the low frequency equivalence principle is consistent with Born scattering. This issue can be quantified further by considering the scalar wave equation (the inhomogeneous Helmholtz equation)

$$
\left(\nabla^{2}+k^{2}\right) \phi(\mathbf{r}, k)=-k^{2} \gamma(\mathbf{r}) \phi(\mathbf{r}, k)
$$

where $\gamma$ is a dimensionless function of space. If $\gamma$ is taken to be of compact spatial support $\mathbf{r} \in V$, then for homogeneous boundary conditions (where $\phi$ and $\nabla \phi$ are zero on the surface of $\gamma$ ), the Green's function solution for a unit amplitude plane wave $\phi_{i}=\exp (i \mathbf{k} \cdot \mathbf{r})$ is given by [38]

$$
\phi(\mathbf{r}, k)=\phi_{i}(\mathbf{r}, k)+k^{2} g(r, k) \otimes \gamma(\mathbf{r}) \phi(\mathbf{r}, k)
$$

where $\otimes$ denotes the (three-dimensional) convolution integral of two functions $f(\mathbf{r})$ and $g(\mathbf{r})$ defined by

$$
g(\mathbf{r}) \otimes f(\mathbf{r})=\int_{\mathbf{r} \in V} g(\mathbf{r}-\mathbf{s}) f(\mathbf{s}) d^{3} \mathbf{s}
$$

and $g(r, k)$ is the Green's function given by

$$
g(r, k)=\frac{\exp (i k r)}{4 \pi r}
$$

An identical solution occurs if $\gamma$ is taken to be an asymptotic potential but where the convolution integral is over all space and not confined to the spatial support $\mathbf{r} \in V$. In either case, Equation (1) is not a solution for $\phi$ and calls for iteration to be applied. The first such iteration gives the Born approximation when

$$
\phi(\mathbf{r}, k)=\phi_{i}(\mathbf{r}, k)+\phi_{s}(\mathbf{r}, k)
$$

where

$$
\phi_{s}(\mathbf{r}, k)=k^{2} g(r, k) \otimes \gamma(\mathbf{r}) \phi_{i}(\mathbf{r}, k)
$$

and $\phi_{s}(\mathbf{r}, k)$ is the Born scattered wave field. The validity of this approximation is predicated on the generic condition that

$$
k^{2}\|g(r, k) \otimes \gamma(\mathbf{r})\|<<1
$$

Thus, the Born approximation becomes an exact solution in the limit as $k \rightarrow 0$. In this context, from Equation (2), we can write

$$
\Phi(\mathbf{r})=\lim _{k \rightarrow 0}\left[\frac{\phi(\mathbf{r}, k)-\phi_{i}(\mathbf{r}, k)}{k^{2}}\right]=\lim _{k \rightarrow 0}\left[\frac{\exp (i k r)}{4 \pi r} \otimes \gamma(\mathbf{r}) \exp (i \mathbf{k} \cdot \mathbf{r})\right]=\frac{1}{4 \pi r} \otimes \gamma(\mathbf{r})
$$


But this asymptotic solution, which assumes that the incident wave field is an ultra-low frequency field, is the same (Green's function) solution as the solution to the Poisson equation

$$
\nabla^{2} \Phi(\mathbf{r})=-\gamma(\mathbf{r})
$$

Thus, it is clear that the Poisson equation, which is the differential form of a Newtonian gravitational field, can be considered to be a description of an exact Born scattered field. In this context, we revisit Newton's law of gravity as given in the following section.

\section{Newton's Law of Gravity Revisited}

The equivalence principle considered in the last section illustrates that, for an observer who can only experience the gradient of a field, there is no way to distinguish between a low frequency wave field generated by a source or a field generated by the source scattering a pre-existing wave field. This principle is fundamental to the interpretation of the generation of a gravitational field given in Case 5 of Figure 1 'Effect of ultra-low frequency scattering with Uber wave'.

For a massive body (taken to be of compact support) consisting of a mass-density $\rho(\mathbf{r})$, the gravitational field generated by a scattering effect is now taken to be given by the space displaced sum of all such point-mass scatterers. The gravitational potential $\phi$ is

$$
\phi(\mathbf{r})=1(\mathbf{r})+\frac{G}{r} \otimes \rho(\mathbf{r})
$$

where $G \simeq 6.674 \times 10^{-11} \mathrm{~m}^{3} \mathrm{~kg}^{-1} \mathrm{~s}^{-2}$ is Newton's Gravitational Constant $(<<1)$. The gradient of this field is given by

$$
\nabla \phi(\mathbf{r})=\nabla 1(\mathbf{r})+\nabla \frac{G}{r} \otimes \rho(\mathbf{r})=\mathbf{0}-\hat{\mathbf{n}} \frac{G}{r^{2}} \otimes \rho(\mathbf{r}) \equiv-\hat{\mathbf{n}} \frac{G}{r^{2}} \otimes \rho(\mathbf{r})
$$

where $\hat{\mathbf{n}}=\mathbf{r} / r$ is a unit vector. This result is of course identical to Newton's model for gravity or equivalently, Einstein's theory of gravity under the weak field approximation when

$$
\phi(\mathbf{r})=\frac{G}{r} \otimes \rho(\mathbf{r})
$$

The difference between Equation (3) and Equation (4) is trivial. However, Equation (3) is based on a low frequency scattering theory for the generation of gravity [39] and is thereby predicated on a causal concept based on an interaction of something with something else, the cause being taken to be the Uber waves generated by the big bang and the interaction being the scattering of such waves with a Higgs Boson. In this context, for a point mass $m_{1} \delta^{3}(\mathbf{r})$ where $\delta^{3}(\mathbf{r})$ is the three-dimensional delta function, then $\rho(\mathbf{r})=m_{1} \delta^{3}(\mathbf{r})$ and Equation (3) becomes

$$
\phi(\mathbf{r})=1(\mathbf{r})+\frac{G}{r} \otimes \rho(\mathbf{r})=1(\mathbf{r})+G \frac{m_{1}}{r}
$$

The acceleration produced by this field is

$$
\nabla \phi(\mathbf{r})=\nabla 1(\mathbf{r})+G m_{1} \nabla \frac{1}{r}=\mathbf{0}-\hat{\mathbf{n}} G \frac{m_{1}}{r^{2}}
$$

so that, for a second mass $m_{2}$, a force will be generated with a magnitude of

$$
F=G \frac{m_{1} m_{2}}{r^{2}}
$$

For the Uber wave scattering theory associated with the schematic for Case 5 in Figure 1, the only difference between this deviation of Newton's law of gravity and the conventional (phenomenological) approach is compounded in the result

$$
\nabla 1(\mathbf{r})=\mathbf{0}
$$


where we note that this specific equation emerges because we have considered a plane wave with a unit amplitude; the result being the same for any constant amplitude. However, it must be understood that the concept under which Newton's law is so derived is through the curvature of space-time by a mass to produce a measurable acceleration given by $\nabla \phi$ where the curvature is the result of ultra-long wavelength space-time waves that are a weak perturbation of a flat space-time.

In the case of Newton's famous formula, as given by Equation (5), the force between the two masses $m_{1}$ and $m_{2}$ is an attractive force. This is because the gravitational potential is created in the negative half-space as illustrated in Figure 1 where the space-time curvature associated with the Uber wave is concave. In the positive half-space, the force is taken to be repulsive, where the space-time curvature associated with the Uber wave is convex and consequently, the space-time remains void of cosmological structures whose formation is predicated on gravity being an attractive force. This concept is illustrated in Figure 2 which shows a single cycle Uber wave field representation for a flat plane instead of a flat space-time fibre as given in Figure 1. The fibre wave representation given in Figure 1 (Case 4) is a Geodesic for this surface taken over the attractive and repulsive gravitational potential components as illustrated in Figure 2. In addition to this surface profile representation, Figure 2 includes the associated contour plots with an equivalent colour code to differentiated between regions of attractive and repulsive gravitational forces. The figure includes an image of a recently discovered massive super cluster of galaxies (the Saraswati supercluster [42]) located approximately 4 billion light-years from Earth which is one of the largest known structures observed in the universe to date. The other image in this figure is an illustration of galaxy superclusters and cosmic voids (the black regions as indicated) [43].

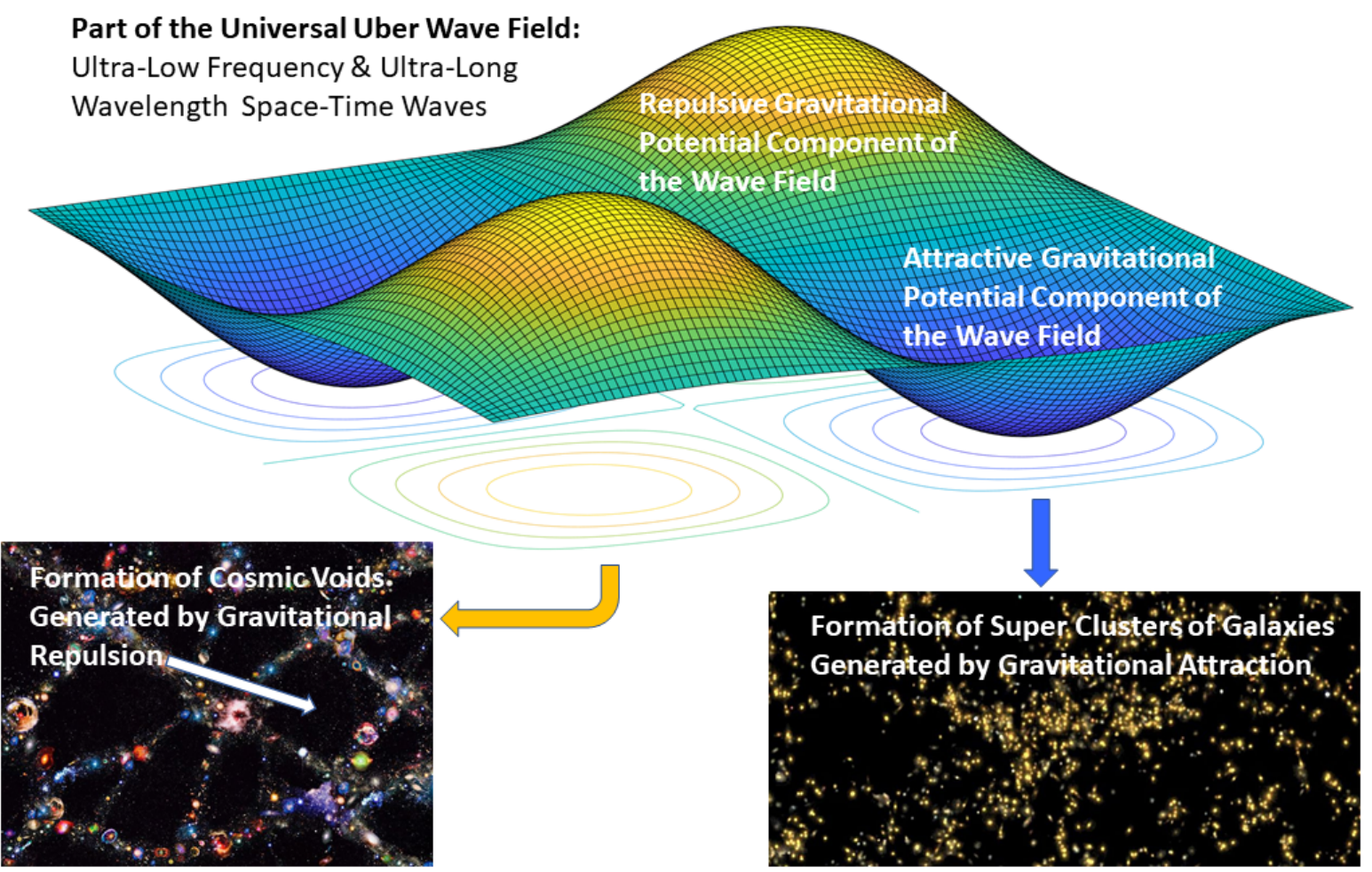

Figure 2. A two-dimensional representation of a (single cycle) Uber wave. The deformation of the surface above and below the common plane represents repulsive and attractive gravitational forces, respectively. The example image to the left illustrates the cosmic voids (as indicated) [43]. The example image on the right hand side shows a super cluster of galaxies [42]. 


\section{Newton's and Einstein's Model for Gravity Revisited: A Basis for the Cosmological Constant}

Newton's model for gravity is compounded in the Equation (4) and is conventionally a phenomenological model providing the 'how' and not the 'why'. In the Uber wave theory, Newton's model is given by Equation (3). For both Equations (3) and (4), the gravitational force $\nabla \phi$ is identical. Thus the Laplacian field $\nabla^{2} \phi$ must also be identical and so for both Equations (3) and (4), we can write Newton's law in terms of Poisson's equation

$$
\nabla^{2} \phi(\mathbf{r})=-4 \pi G \rho(\mathbf{r})
$$

which has the Green's function solution that is Equation (4).

Einstein's field equations (from the theory of general relativity) are given by [5]

$$
G_{\mu \nu}=\frac{8 \pi G}{c_{0}^{4}} T_{\mu \nu}
$$

where $G_{\mu \nu}$ is the Einstein tensor, $T_{\mu \nu}$ is the stress-energy tensor, the summation over the indices $\mu$ and $\nu$ being implied in terms of both indices ranging over $0,1,2,3$ corresponding to $c_{0} t, x, y, z$, respectively. Comparing Equation (6) with Equation (7) we see that, in the latter case, the source function is an energy-momentum tensor (which includes both mass-densities and currents) and the gravitational potential is replaced with a metric tensor.

When the gravitational field is weak and the sources are moving very slowly compared to light-speed, Equation (6) becomes a scalar field approximation to Equation (7), the metric tensor having been expanded in terms of the gravitational potential. In this case (the weak field approximation), Equation (7) describes wave behaviour which can be examined analytically by considering a flat (Minkowski) space perturbed by a wave amplitude metric denoted by $h^{\alpha \beta}$. This is the case when a source is taken to be generating gravitational waves (an accelerating mass) which is far from the point in space-time at which the gravitational waves are observed; where the gravitational field is weak and the space-time curvature approximates that of a Minkowski space. Since, conventionally speaking, space-time is flat in the absence of a gravitational field, a weak gravitational field can be defined as one in which space-time is 'nearly' flat. This approximation linearises Equation (7) and yields the inhomogeneous wave equation

$$
\partial^{\alpha} \partial_{\alpha} h^{\alpha \beta}=\frac{16 \pi G}{c_{0}^{4}} T^{\alpha \beta}
$$

which, for an empty space-time has the homogeneous form $\partial^{\alpha} \partial_{\alpha} h^{\alpha \beta}=0$.

By induction, and, through a comparison of Equation (3) with Equation (4), for Equation (7) to become compatible with the Uber wave theory being proposed, it should be replaced with the field equation

$$
G_{\mu \nu}+\Lambda g_{\mu \nu}=\frac{8 \pi G}{c_{0}^{4}} T_{\mu \nu}
$$

where $g_{\mu \nu}$ is the metric tensor and $\Lambda$ is the cosmological constant. The introduction of this constant was originally considered by Einstein in order to maintain a static universe. He then considered it to be erroneous when the universe was understood to be expanding and not static. Here, it is re-introduced, not to model a static universe, but an expanding universe under going an acceleration through dark energy, an effect that can now be understood in terms of the Uber wave concept being proposed.

\section{Some Example Consequences}

Some of the consequences of the theory of gravity compounded (schematically) in Figure 1 and Figure 2 are as follows:

(i) The negative half-space of Figure 1 represents a gravitational zone where light is curved in a convergent sense leading to the generation of Einstein rings, for example [40], [41]. These zones are therefore analogous to the effect of a convergent lens, a convergence that occurs due to the concave 
gravitational field surrounding massive bodies but also (at least on a larger cosmological scale) when mass is not present (dark matter).

(ii) In the positive half-space of Figure 1, light and other electromagnetic waves will be curved in a divergent sense. Einstein rings can not be generated because stars and galaxies can not exist. However, in this half-space, at least on a cosmological scale, the divergence of light may occur anyway. In terms of their effect on the propagation of light, these zones are analogous to a divergent lens.

(iii) The nature of gravity in the positive half-space of Figure 1 implies that if we are in the line of sight of a cosmic void, we would not be able to see any galaxy cluster behind the void generated in the neighbouring concave space-time curvature associated with another cycle of an Uber wave. Thus, there could be luminous super clusters in the universe that can never be observed.

(iv) Images of the universe obtained by divergent light could be miss-interpreted due to a cosmic mirroring effect (by analogy with the effect of a mirror reflecting light), where light is obtained that has undergone significant divergence or reflection from an anti-gravity cosmic void. This is an effect that might be observable by searching for cosmic structures that appear to be identical (subject to some geometric distortion) when observed from different angles in the sky, especially images of galaxy clusters that exist close to cosmic voids. This leads to the idea that many cosmic structures are not in the positions of the sky that they appear to be and further, that there is more observable luminescent matter than actually exists, at least the matter that is not hidden behind cosmic voids.

(v) If we traveled from the negative to positive half-space in a space ship, for example, we would be pushed away from the positive half space noting that in the transition from one to the other where the amplitude of the Uber wave is zero, there will be no gravity at all. If the space ship was very large (the size of a planet say with a mass that is effected by gravity), it would be ripped apart when it entered the positive half-space. However, the remaining matter (the clumps of matter small enough not to be influence by gravity) would still generate an increase in the homogenous mass density of this half-space.

(vi) If a massive or super-massive black hole (which can only be formed in the negative half-space) was to enter the positive half-space, then it also would be ripped apart releasing its previously unseen mass (and information content) into this half-space, thereby significantly contributing to the mass density and thus to the anti-gravitational forces of a cosmic void. Such black hole explosions would be through an entirely different mechanism to the explosions associated with the production of Hawking radiation [46]. This concept has consequences in regard to the black hole information paradox [44].

(vii) Taking cosmic voids to be the source of gravitational repulsion, one might expect them to disproportionately 'compress' those regions of the universe containing superclusters in respect of, and, in addition to their effect on the expansion of the universe. This could account for the stringiness and striations of superclusters associated with the formation of the cosmic web as illustrated in Figure 2, i.e. the image illustrating the formation of cosmic voids. Thus, as well as increasing the rate of expansion of the universe, cosmic voids would disproportionately compress those regions of space composed of luminous matter, i.e. the cosmic web is a consequence dark energy compressing the 'by-products' of dark matter.

(viii) In Figure 1, the schematic single cycle Uber wave is taken to oscillate very slowly over cosmic time scales while the space-time that supports it, expands according to Hubble's law, an expansion that is further accelerated through the effect of dark energy attributed to the cosmic voids. If the wave is taken to be part of a larger wave complex, then the polarities of the amplitudes (which are representative of a demarcation between attractive and repulsive gravitational forces) will 
eventually reverse. Over time, this would lead to a transformation and indeed a 'reversal' of the cosmic void/web structure in which the universe oscillates between the creation, destruction and re-creation of luminance matter as the Uber wave complex oscillates over cosmological time scales. This is a very different scenario to the current theoretical ideas relating to the ultimate fate of the universe such as the 'Big Freeze or Heat Death', and the Big 'Rip', 'Crunch', 'Bounce' and 'Slurp', for example, each possibility being based on a very simple form for the dark energy equation of state [47].

\section{Generation of Complex Patterns through Wave Field Interactions}

The Uber wave field patterns illustrated in Figure 1 and Figure 2 are, as discussed before, highly simplified schematic representations of such waves in terms of their physical characteristics and scale. These figures are also entirely incompatible with the complexity of the patterns that Uber waves would be expected to develop. Complex wave field patterns can easily be produced by allowing a wave field, initially generated by a small number of point sources, to continually propagate, reflect and interfere. An example simulation of such a complex wave pattern is given in [48] which provides a simulation based on the two-dimensional classical scalar wave equation for a square but flexible surface starting with a very simple initial conditions. The resulting waves spread, interfere and mix (without energy loss) to give a complex rippled surface. The purpose of this simulation is to show how relatively simple it is to generate complex images that are textually consistent with the measured patterns of the cosmic microwave background radiation [49], for example; a texture that can be quantified in terms of its variable fractal dimension and other multi-fractal parameters of such images (e.g. [50] and [51]).

The texture associated with the Wilkinson Microwave Anisotropy Map (WMAP) image is based on the propagation of electromagnetic waves which are self-propagating waves and do not require a medium of propagation. This is because the oscillation of an electric field yields a magnetic field which oscillates generating another cycle of the electric field and so on, i.e. each electric and magnetic field component creates the other in a succession that leads to the propagation of an electromagnetic wave. However, in the Uber wave theory presented here, the wave field does require a medium of propagation which is space-time itself. Nevertheless the propagation and interaction of Uber waves may be considered in a way that is analogous to that of microwaves and may be expected to generate similar complex and possibly self-affine textures, e.g. the textures associated with cosmic voids when 'observed' over a universal scale. The scattering of such waves with matter will not in itself, produce complex wave patterns. This is because the frequency of scattering events take place over a scale in which the frequency is effectively zero. In this sense, a star is a near-perfect Born scatterer. However, the interaction of billions of such point scatterers (associated with the scale being considered) subject to the influence of the gravitational force due to dark matter, will produce a multitude of complex structures that is compounded in the observable universe. Coupled with the disproportionate compression of the space-time containing these complex structures by cosmic voids, one would expect a texture to develop that is more consistent with the cosmic web structure of the observable universe than the textures associated with the WMAP.

\section{Commentary on the Concept}

The purpose of this exploratory paper has been to consider a conceptual model to explain dark matter and dark energy. This has been undertaken within the context of a linearised version of Einstein's equations when gravitational waves become a natural and self-consistent physical concept. The underlying approach is to consider a wave field model and attempt to explain the field models that emerge. For the development of a deeper understanding of physics, this has one primary advantage; it transcends the phenomenology associated with an assigned property producing a field such as charge generating an electric field, and, within the context of this work, a mass producing a gravitational field through the curvature of space-time.

The field equations of physics always tend to out rank the wave field equations in terms of a cause and effect scenario, standard examples of this being Maxwell's equations [52] for an electromagnetic field and Einstein's equations for a gravitational field [5]. But this is not always the case. For example, the Proca-Maxwell equations [53] are a modification of Maxwell's equations so that they conform to 
the relativistic Klein-Gordon wave equation instead of the classical (non-relativistic) wave equation [54]. This conformity leads to a set of equations (in differential form) where Coulombs law and Amperes law (with Maxwell's 'displacement term') include extra terms involving the mass (squared). This yields a mathematical description of Vector Bosons which have a spin of 1 . These Bosons include the experimentally verified $W$ and $Z$ particles which provide the basis for a unified theory to describe the electromagnetic and weak forces. These Bosons interact with a Higgs Boson which is the only known Boson to be represented by a scalar field (the scalar wave field associated with the Klein-Gordon equation) with a spin of zero.

In the same way, it may be argued that the Dirac field equations are a manifestation of the Klein-Gordon equation which conserves the probability current density, thereby conforming with the non-relativistic case (i.e. Schrödingers equation [55]) and in doing so, introducing the concept of (scalar) spinor fields where particles such as an electron are examples of Fermions with a spin of 1/2 [56], [57]. In regard to this development, it is worth noting that in 1941 an identical approach was taken by Rarita and Schwinger [58] in which Einstein's field equations were modified so that upon linearisation, they conformed to the Klein-Gordon rather than the classical wave equation. This led to the idea that there should be a class of particles with a spin of $3 / 2$ that generate vector spinor fields although to date, no such particles have been discovered experimentally. In each case, the Dirac, Proc-Maxwell and Rarita-Schwinger field equation are derived by making existing field equation conform to a governing wave field equation. In this context, the approach considered in this paper has been to modify the interaction of an assumed gravitational wave field with a mass so that an accepted gravitational field model is obtained, at least within the weak field approximation of the theory of general relativity. This is based on the low-frequency limiting condition and the equivalence principle presented in Section 5 that is applicable under this condition.

In a more general context, the conceptual model considered in this paper is an example of taking a stationary model to be a limiting case of a dynamic effect. However, it is important to understand that the model is not based on assuming time independence. Rather, it is based on the idea that if the wavelength of a wave field is so large compared to the scale of a scatterer, then in the spatial location of the scatterer, there is no apparent wave motion, thereby giving the appearance of a time independent effect. This 'effect' then becomes the asymptotic result obtained when the frequency of the wave field approaches zero. An Uber wave field may be a very slowly varying wave field but it still propagates at a finite velocity less than or equal to light speed. In this respect, Newton's law of gravity only appears to be a time-independent model. If we consider a fundamental axiom of all physics to be that nothing, absolutely nothing remains the same but evolves in time, then it is the original conceptual basis of Newton's law that, when weighed in the balance is found wanting! As discussed in Section 5, the only difference between the two concepts, is compounded in the gravitational potential being described with or with out, what is in effect, a cosmological constant determined by the amplitude of a near zero frequency Uber wave field.

One of the reasons why gravitational waves generated by cosmic sources are so weak and therefore so difficult to measure, is related to the stiffness of space-time. Measured in units of $\mathrm{GPa}$ or $\mathrm{kN} \mathrm{mm}^{-2}$, the Elastic Modulus (the stiffness) of Steel is approximately 200, that of a Diamond, 1200, while Graphene has a stiffness of 1TPa, for example. Steel, Diamond and even Graphene are materials that support mechanical waves and as the material becomes stiffer, the greater the energy that is required to propagate mechanical waves through the material. If we think of space-time in terms of a 'material' (by analogy only) that supports the propagation and scattering of space-time waves, it is possible to develop an analogous value for the stiffness or rigidity of space-time in terms of the Young's Modulus. Given that Equation (7) can be loosely interpreted as

\section{Curvature of spacetime $=$ Constant $\times$ Energy and Momentum}

then the Constant $\left(=8 \pi G / c_{0}^{4} \sim 2 \times 10^{-43} \mathrm{~s}^{2} \mathrm{Kg}^{-1} \mathrm{~m}^{-1}\right)$ determines how easily the energy-momentum tensor can curve space-time. Since this constant is so small, it is clear that any 'deformation' in space-time requires a significant amount of energy and momentum and vast energy sources to create measurable space-time wave fields. In the 2015 event [32], where the source of the gravitational waves measured was due to the merger of two black holes, the total energy emitted was $\sim 2 \times 10^{41} \mathrm{kWh}$ and the peak luminosity was $3.6 \times 10^{49} \mathrm{~W}$ which (at least for an instant in time) was more than the luminosity of all the stars in the observable universe $\left(\sim 10^{48} \mathrm{~W}\right)$.

Attempts to evaluate Young's modulus for the fabric of space-time has been considered in [59] and [60], for example, by making simple analogies between the strength of materials and Equation (7) leading to a 
Young's Modulus of $\sim 10^{113} \mathrm{Nm}^{-2}$. Following the discovery of gravitational waves in 2015, the question has arisen as to what is the Young's modulus $Y$ of space-time as experienced by space-time waves which is compounded in the result $Y \sim c_{0}^{2} f^{2} / G \sim 10^{27} f^{2}$ where $f$ is the frequency of the space-time wave [61]. On the other hand, further arguments on the grounds that a Young's modulus must be limited by the energy density of the matter through which a gravitational wave propagates, yields an upper limit for this modulus which is quantified by the result $Y<2.5 \times 10^{-17} c_{0}^{2} f^{2} / G \sim 3.4 \times 10^{10} f^{2}[62]$.

The stiffness of space-time means that it is not easy to deform. This is why it takes such a large mass to generate a relatively small curvature of space-time, whose gradient generates a measurable gravitational force. Similarly, a space-time wave can only deform space-time by a relative small amount. Thus, it is for this reason that, from the point of view of developing a theoretical model for space-time Uber wave, we consider them to be propagating in a Minkowski space in which the deformation of space-time is small; a weak perturbation of a 'flat space-time'. In regard to the conceptual model described in this paper, it is assumed that the Uber waves conform to a Minkowski space with concave and convex deformations to space-time that occur over wavelengths on a cosmic scale. This leads to an inconsistency in that we are using a linear concept compatible with the linearisation of a non-linear field equation (Einstein's equations) in order to describe the generation of a field that is best described by the non-linear field equation in the first place. How can a linear based wave model yield a nonlinear field equation?

What is required is to develop the approach in such a way that Einstein's field equations emerge from an analysis of a tonsorial gravitational wave field interaction with a Higgs field. On the other hand, if a massive body such as a black hole develops, which is taken to be due to a build up of mass over time due to the interaction of an Uber wave with an increasingly large Higgs field in a Minkowski space (or is generated suddenly in the aftermath of a Supernover, for example), there is nothing intrinsically wrong with the idea that, in this case, Einstein's equations become required to define the strong gravitational field that is created. In this case, instead of a non-linear 'deformation' of space-time reducing to the linear case, we need to consider a linear deformation of space-time to undergo an 'accretion' to give a non-linear representation, namely, Einstein's gravitational field equations. This is because Einstein's equations are required to represent the gravitational field of a Black Hole; a linear representation is not sufficient. The argument for this is that because space-time is so stiff, the majority of Uber wave scattering can be taken to occur in a Minkowski space, except when gravitational singularities occur. This is an example of adopting a linear wave field theory to produce a localised nonlinear field and has been studied in regard to acoustics and the evolution of deep sea surface freak waves, for example. A phenomenological approach to this problem is to consider the non-linearisation of a linear equation, namely, the non-linear Schrödinger equation which is an otherwise linear field equation [63].

In the context of acoustics and fluid dynamics, gravitational waves have a first order synergy with acoustic waves given that they have a compressional space-time component which is in the direction of propagation as is the compression and rarefaction of the pressure field in an acoustic wave. In terms of Figure 1 (Cases 4-6), we can consider an analogy from acoustics where the positive half-space is the compressional component and the negative half-space is the associated rarefaction. Let both sections of the wave become effectively frozen in time (due to the ultra-low frequency of the oscillation) but where the molecular motions in both components of the wave continue. In this non-adiabatic hypothetical scenario, the pressure in the positive half-space (relative to the negative half-space) will cause the extent of the half-wavelength in the positive half-space to increase and the half-wavelength in the negative half-space to decrease. In this context, the compression component will elongate relative to the extent of the rarefaction. This thought experiment provides an acoustical analogy to understanding the evolution of cosmic voids through the 'dark energy effect', an evolution that compresses (with an apparent acceleration) the dark matter regions of space-time into a filamentary complex as illustrated schematically through Figure 2 in a conceptual explanation of the cosmic web.

\section{Summary}

In terms of developing an understanding of dark matter and energy, the basic idea is compounded in Figure 1 and the explanation thereof on the case-by-case basis given in Section 4. This is a conceptual model and its interpretation is not taken to be proven theoretical or validated experimentally. It is merely an attempt to explain physical effects that currently exist in name only through the assumption that a 
gravitational field is the by-product of cosmic wave interactions with a Higgs field at the low-frequency limit of the big bang spectrum when space-time Uber waves are taken to be first generated. The key to this interpretation is the equivalence principle discussed in Section 5 in which, using a scalar field model, a gravitational force generated by a low frequency scattering interaction is indistinguishable from the force associated with a conventional phenomenological model for gravity. In this context, when readers next observe a point of light in the night sky (i.e. a star or planet, for example), they might think upon it as being a near perfect point scatterer of cosmological space-time waves generated at the creation of the universe itself, i.e. a manifestation of an exact Born scattering interaction. In this sense, and, in the context of the concept proposed, any star or other observable feature in the night sky is a testament to the big bang itself.

\section{Conclusion}

The conceptual model proposed in this paper yields the following possible explanations for dark matter and dark energy:

What is dark matter? It is the background gravitational field associated with the component of an Uber wave field in which space-time undergoes a concave curvature and where luminous matter may develop due to gravity being an attractive force.

What is dark energy? It is the background gravitational field associated with the component of an Uber wave field in which space-time undergoes a convex curvature, where luminous matter can not form because gravity is a repulsive force and why cosmic voids are formed with a low and homogenous mass density. However, the mass density is still large enough to generate an accelerative expansion of the observable universe as the voids get larger, and, in the process, produce cosmic web structures of luminous matter that is 'under the control' of dark matter.

The key to these explanations is compounded in a model for the fabric of space-time which is not assumed to be a flat fabric but a fabric supporting waves, waves oscillating at frequencies that, in the context of conventional cosmological scales, are effectively zero, but are waves nonetheless. The principal differences between these models are quantified schematically in Case 1 and Case 4 of Figure 1. The concept has been explored in terms of a schematic relating to a single fibre of space-time and further qualified using a scalar wave field model, i.e. a Newtonian field model under the guise of the weak field approximation. However, the same principles can be established by developing a tensor field theory for the propagation and scattering of space-time Uber waves, i.e. a tensorial wave field scattering theory. Finally, it is noted that the particulate characteristics of an Uber wave (in a quantum mechanical context) might be interpreted in terms of a so-called 'Graviton' which is, through an inference associated with the concept presented in this paper, a massless particle.

Acknowledgements. The author acknowledges the support of the Science Foundation Ireland; the School of Electrical and Electronic Engineering, Technological University Dublin; the School of Mathematics, Statistics and Computer Science, University of KwaZulu-Natal; the Faculty of Natural Science, University of Western Cape and Wrexham Glyndwr University of Wales. The author is grateful for the contributions made through dialogue and correspondence with Mr Mark Blackledge, Dr Keith Hopcraft, Prof Alison McMillan, Dr William Rawes and Prof Michael Rycroft.

\section{References}

1. F. Zwicky, "Die rotverschiebung von extragalaktischen nebeln," Helvetica Physica Acta, vol. 6, pp. 110-127, 1933.

2. F. Zwicky, "On the masses of nebulae and of clusters of nebulae," Astrophysical Journal, vol. 86, p. 217, 1937.

3. E. A. Hubble, "Relation between distance and radial velocity among extra-galactic nebulae", Astronomy, vol. 15, pp. 168-173, 1929. Available: https://www.pnas.org/content/pnas/15/3/168.full.pdf 
4. G. Lemaître, "A homogeneous universe of constant mass and increasing radius accounting for the radial velocity of extra-galactic nebulae," Annales de la Société Scientifique de Bruxelles, vol. 47, pp. 49-59, 1927. Available: http://www.physics .umd.edu/grt/taj/675e/Lemaitre1927.pdf

5. A. Einstein, "The foundation of the general theory of relativity," Annalen der Physik, vol. 49, pp. 770-822, 1916.

6. A. G. Riess et al., "Observational evidence from supernovae for an accelerating universe and a cosmological constant," Astronomical Journal, vol. 116, no. 3, pp. 1009-1038, 1998.

7. P. J. R. Peebles and R. Bharat, "The cosmological constant and Dark Energy," Reviews of Modern Physics vol. 75, no. 2, pp. 559-606, 2003.

8. K. Griest, "The search for the dark matter: WIMPs and MACHOs,". Annals of the New York Academy of Sciences. vol. 688, pp. 390-407, 1993.

9. M. Kamionkowski, "WIMP and axion dark matter". High Energy Physics and Cosmology, The ICTP Series in Theoretical Physics, World Scientific Publishers, vol. 14:, pp. 394-412, 1997. Available: https: //arxiv.org/pdf/hep-ph/9710467.pdf

10. A. K. Drukier and K. Freese and D. N. Spergel, "Detecting cold dark-matter candidates", Physical Review D, vol. 33, no. 12. pp. 3495-3508, 1986.

11. K. Freese, J. Frieman and A. Gould, "Signal modulation in cold dark matter detection", Physical Review D, vol. 37, no. 12 , pp. 3388-3405, 1986.

12. J. H. Davis, "The past and future of light dark matter direct detection". Int. J. Mod. Phys. A, vol. 30, no. 15) 1530038. arXiv:1506.03924, 2015.

13. E. Del Nobile, G. B. Gelmini, P. Gondolo and J. Huh, Ji-Haeng, "Update on the halo-independent comparison of direct dark matter detection data", Physics Procedia, vol. 61, pp. 45-54, 2015.

14. G, Bertone, "The moment of truth for WIMP dark matter", Nature, vol. 468, pp. 389-393, 2010.

15. G. Bertone, "Particle dark matter: Observations, models and searches", Cambridge University Press, 2010. ISBN 978-0-521-76368-4

16. G. Angus, G. (2013). "Cosmological simulations in MOND: The cluster scale halo mass function with light sterile neutrinos", Monthly Notices of the Royal Astronomical Society, vol. 436, no. 1, pp. 202 ?211. arXiv:1309.6094.

17. M. Jamil, K. Yesmakhanova, D. Momeni, R. Myrzakulov, "Phase space analysis of interacting dark energy in $f(T)$ cosmology", Cent. Eur. J. Phys., vol. 10, no. 5, 1065-1071, 2012. DOI: 10.2478/s11534-012-0103-2

18. S. Capozzielo, R. D'agostino and O. Luongo, "Extended gravity cosmography", International Journal of Modern Physics D, World Scientific Publishing, vol. 28, no. 10, 1930016, 2019. Available: https://www. researchgate.net/publication/332197305_Extended_Gravity_Cosmography

19. W. Hu and S. Dodelson, "Cosmic microwave background anisotropies", Ann. Rev. Astron. Astrophys., vol. 40, pp. 171-216, 2002.

20. A. Einstein, "Kosmologische betrachtungen zur allgemeinen relativitätstheorie". Sitzungsberichte der Königlich Preussischen Akademie der Wissenschaften. Berlin, DE. Part 1, pp. 142?152, 2017.

21. L. Dyson, M. Kleban and L. Susskind, L. (2002). "Disturbing implications of a cosmological constant". Journal of High Energy Physics, vol. 10, p. 011, 2002. Available: https://iopscience.iop.org/article/ 10.1088/1126-6708/2002/10/011/pdf

22. G. R. Blumenthal, S. M. Faber, J. R. Primack and M. J. Rees, "Formation of galaxies and large-scale structure with cold dark matter", Nature, vol. 311, no. 517, pp. 517?525, 1984.

23. M. Chojnowski, "Dark matter model from the idea of multi-cohesive areas", Canadian Journal of Physics, vol . 95, no. 10, 2017. Available: https://doi.org/10.1139/cjp-2017-0144

24. International Journal of Modern Physics D, Gravitation; Astrophysics and Cosmology, World Scientific, ISSN (online): 1793-6594. Available: https://www.worldscientific.com/worldscinet/ijmpd

25. Special Issue "Focus on dark matter", MDPI Special Issue of Universe (ISSN 2218-1997), Section 'Cosmology', 2020-2021. Available: https://www.mdpi.com/journal/universe/special_issues/focus_dark_matter

26. F. W. Dyson, A. S. Eddington and C. Davidson, "A determination of the deflection of light by the sun's gravitational field from observations made at the total eclipse of May 29, 1919". Philosophical Transactions of the Royal Society A: Mathematical, Physical and Engineering Sciences, vol 220 (571?581), pp. 291?333, 1920. Available: https://royalsocietypublishing.org/doi/10.1098/rsta.1920.0009

27. J. Earman and C. Glymour, "Relativity and eclipses: The British eclipse expeditions of 1919 and their predecessors", Historical Studies in the Physical Sciences, vol. 11, no. 1, pp. 49-85, 1980.

28. M Strevens, The knowledge machine: How irrationality created modern science, Liveright Publishing Corporation, 2020. ISBN: 9781631491375

29. K. T. McDonald, "Laplace and the speed of gravity", 2018. Available: https://www.physics.princeton. edu/ mcdonald/examples/laplace.pdf

30. J. A. Wheeler and K. W. Ford, "Geons, black holes, and quantum foam: A life in physics," W. W. Norton \& Company, 2003. ISBN: 0393319911 
31. P. Higgs, "Broken symmetries and the masses of gauge bosons", Physical Review Letters, vol. 16, 508-509, 1964.

32. B. P. Abbott et al., "Observation of gravitational waves from a binary black hole merger," Physical Review Letters (American Physical Society), vol. 116, 061102, 2016. Available: https://physics.aps.org/ featured-article-pdf/10.1103/PhysRevLett.116.061102.

33. M. H. Poincaré, "Sur la dynamique de l' électron," Institute of France, Académie des Sciences pp. 1504-1508, 1905. Available: https://www.academie-sciences.fr/pdf/dossiers/Poincare/Poincare_pdf/Poincare_ CR1905.pdf

34. A. Einstein, "Über Gravitationswellen," Sitzungsberichte der Königlich Preussischen Akademie der Wissenschaften (Berlin), pp. 154-167, 1918.

35. A. Einstein and R. Nathan, "On gravitational waves," Journal of the Franklin Institute, vol. 223, no. 1, pp. 43-54, 1937.

36. J. M. Blackledge, "On the chirp function, the chirplet transform and the optimal communication of information", IAENG International Journal of Applied Mathematics, vol. 50, no. 2, pp. 285-319, 2020. Available: https://arrow.tudublin.ie/engscheleart2/218/

37. M. Born, "Quantenmechanik der stossvorgänge," Zeitschrift für Physik, vol. 38, pp. 803-827, 1926. Available: https://link.springer.com/article/10.1007/BF01397184

38. G. Evans, J. M. Blackledge and P. Yardley, Analytic solutions to partial differential equations, 1999, Springer. ISBN: 2540761241

39. J. M. Blackledge, "Gravity as an ultra-low frequency scattering effect," Mathematica Eterna, vol. 6, no. 3, 467-480, 2016. Available: https://arrow.tudublin.ie/engscheleart2/106/

40. A. Einstein, "Lens-like action of a star by the deviation of light in the gravitational field," Science, vol. 84, no. 2188, pp. 506-507, 1936.

41. J,. Renn, T. Sauer and J. Stachel, "The origin of gravitational lensing: A postscript to Einstein's 1936 science paper.," Science vol. 275, no. 5297, pp. 184-186, 1997.

42. P. Dockrill, "This dizzying galaxy supercluster will change your perspective on the cosmos," Science Alert, 22 July, 2018. Available: https://www.sciencealert.com/scientists-discover-one-of-the-largestknown-structures-in-the-universe-Saraswati-galaxy-supercluster

43. C. Gearin, "BOSS supercluster," 11 March, 2016. Available: https://www.bibliotecapleyades.net/ universo/cosmos222.htm

44. S. Hossenfelder, "How do black holes destroy information and why is that a problem?", Back Reaction August 23, 2019. Available: http://backreaction.blogspot.com/2019/08/ how-do-black-holes-destroy-information.html

45. S. W. Hawking, "Particle creation by black holes," Communication in Mathematical Physics, vol. 46, pp. 206-206, 1976.

46. S. W. Hawking, "Black hole explosions," Nature, vol. 248, pp. 30-31, 1974.

47. F. Adams and L. Gregory, The five ages of the universe: Inside the physics of eternity, Simon and Schuster, Australia. 2000. ISBN 978-0-684-86576-8.

48. W. Rawes, "Classical wave model mixes making a pattern similar to the measured cosmic microwave background", YouTube, 2019. Available: https://www . youtube.com/watch?v=msjRLt1zJB4\&app=desktop

49. C. L. Bennett et al., "Nine-year wilkinson microwave anisotropy probe observations: Final maps and results," Astrophysical Journal Supplement vol. 208, no. 2, p. 20, 2013.

50. M. J. Turner, J. M. Blackledge and P. R. Andrews, Fractal geometry in digital imaging, Academic Press, 1998. ISBN-10: 0127039708

51. J. S. Bagla, J. Yadav and T. R. Seshadri, "Fractal dimensions of a weakly clustered distribution and the scale of homogeneity," Monthly Notices of the Royal Astronomical Society, vol. 390, no. 2, 2008. Available: https://ieeexplore.iee. org/abstract/document/8219974

52. J. C. Maxwell, "A dynamical theory of the electromagnetic field," Philosophical Transactions of the Royal Society of London, vol. 155, pp. 459-512, 1885.

53. A. Proca, "Fundamental equations of elementary particles.", C. R. Acad. Sci. Paris, vol. 202, no. 1490, 1936.

54. W. Greiner, Relativistic quantum mechanics, Edition 3, Springer, 2000.

55. E. Schrödinger, "Quantization as an eigenvalue problem," Annalen der Physik, vol. 489, no. 79, 1926.

56. P. A. M. Dirac, "The quantum theory of the electron, Part 1," Proc. R. Soc. (London) A, vol. 117, pp. 610-612, 1928

57. P. A. M. Dirac, "The quantum theory of the electron, Part II," Proc. R. Soc. (London) A, vol. 118, pp. 351-361, 1928.

58. W. Rarita and J. Schwinger, "On a theory of particles with half-integral spin," Phys. Rev., vol. 60, pp. 61-69, 1941. 
59. T. G. Tenev and M.F. Horstemeyer, "Mechanics of space-time: A solid mechanics perspective on the theory of general relativity," Int. J. Mod. Phys. pp. 1-30, 2016. Available: https://arxiv.org/vc/arxiv/papers/ 1603/1603.07655v1.pdf

60. D. Izabel, "Can we estimate the Young's modulus of space-time from simple analogies based on the concepts of the strength of Mmaterials? Reflection and proposals," HAL Archives-Ouvertes, HAL Id: hal-01562663, pp. 1-11, 2017. Available: https://hal.archives-ouvertes.fr/hal-01562663/document

61. K. T. McDonald, "What is the stiffness of spacetime?", 2018. Available: http://physics.princeton.edu/ $\sim$ mcdonald/examples/stiffness.pdf.

62. A. C. Melissinos, "Upper limit on the stiffness of space-time", 2018. Available https://arxiv.org/pdf/1806. 01133.pdf

63. J. M. Blackledge, "A generalised nonlinear model for the evolution of low frequency freak waves," International Journal of Applied Mathematics, vol. 41, no. 1, pp. 33-55, 2010. Available: https://arrow.tudublin.ie/ engscheleart $2 / 10$ 